\title{
Correspondence
}

\section{Eliminating tuberculosis by 2035: tackling the financial barriers at all stages of the cascade of care}

We welcome the recent paper by Pedrazzoli et al. ${ }^{1}$ that discusses how to measure the economic burden for tuberculosis (TB) patients, and outlines how this information can inform policies for better social and financial protection. The current methodology, however, only includes TB patients enrolled and retained in care. While the paper mentions the need to assess patients who are lost to follow-up between diagnosis and treatment (pre-treatment loss to follow-up), or during treatment, we feel that earlier aspects of the cascade of care also need emphasis-especially if we are to make the goal of eliminating TB by 2035 a reality.

Two recent studies in Zimbabwe highlight the huge pre-diagnostic losses to follow-up that can occur between onset of symptoms, when patients are identified as having presumptive $\mathrm{TB}$, and laboratory diagnosis. ${ }^{2,3}$ The first study found that $55 \%$ of HIVinfected persons with presumptive TB who should have had a diagnostic Xpert ${ }^{\circledR}$ MTB/RIF assay (Cepheid, Sunnyvale, CA, USA) failed to have the test done, ${ }^{2}$ and the second study conducted in Guruve (a rural district) found that $19 \%$ of nearly 3000 persons with presumptive TB failed to be investigated in a district laboratory. ${ }^{3}$ In Guruve, an important risk factor was distance between the health facility that registered the presumptive $\mathrm{TB}$ patients and the laboratory tasked with analysing the specimens. Other risk factors for pre-diagnostic loss to followup include TB-related stigma, direct non-medical costs (including food and transport), income loss and direct medical costs, including payments for laboratory tests, consultation fees and hospitalisation. These costs are compounded by long health service waiting times and the large numbers of clinic visits before a diagnosis is made. Persons with presumptive TB may also have comorbidities (such as HIV), which can further complicate and delay diagnosis, especially when sputum smear microscopy and chest radiography are the only available diagnostic tools in that catchment area. It is not surprising that financial resources in poverty-stricken households can be exhausted long before a definitive diagnosis of TB is made.

As the Lancet Commission on building a TB-free world emphasises, more attention needs to be paid to implementing and integrating patient pathway analyses into national TB programme activities so that there is a far better understanding of how and where persons with $\mathrm{TB}$ symptoms seek care and where people fall out of the care cascade. ${ }^{4}$ Resources can then be reallocated to where they are really needed to identify the missing TB cases. Social protection mechanisms for low-income countries, such as cash transfers, transport reimbursement vouchers or social health insurance schemes that are being tested in middle-income, high TB burden countries in Asia, ${ }^{5}$ can be evaluated to determine whether they help in reducing losses to follow-up at all stages of the care cascade. The second pillar of the WHO End TB Strategy emphasises bold policies and supportive systems around social protection, poverty alleviation and other determinants of $\mathrm{TB}$, and these must lead to bold actions to ensure that no one with TB is left behind.

C. TIMIRE ${ }^{1,2}$

C. SANDY ${ }^{2}$

A. D. Harries ${ }^{1,3}$

${ }^{1}$ International Union Against Tuberculosis and Lung

Disease

Paris, France

${ }^{2}$ Ministry of Health and Child Care

National AIDS \& TB programme

Harare, Zimbabwe

${ }^{3}$ Department of Clinical Research

Faculty of Infectious and Tropical Diseases

London School of Hygiene \& Tropical Medicine London, UK

e-mail:collinstimire2005@yahoo.com http://dx.doi.org/10.5588/ijtld.19.0248

Conflicts of interest: none declared.

\section{References}

1 Pedrazzoli D, Borghi J, Viney K, et al. Measuring the economic burden for TB patients in the End TB Strategy and Universal Health Coverage frameworks. Int J Tuberc Lung Dis 2019; 23: 511.

2 Takarinda K C, Choto R, Sandy C, et al. How well does the process of screening and diagnosis work for HIV-infected persons identified with presumptive tuberculosis who are attending HIV care and treatment clinics in Harare city, Zimbabwe? Trans R Soc Trop Med Hyg 2018; 112: 450-457.

3 Murongazvombo A S, Dlodlo R A, Shewade H D, et al. Where, when and how many tuberculosis patients are lost from presumption until treatment initiation? A step by step assessment in a rural district in Zimbabwe. Int J Infect Dis 2018; 78: 113-120.

4 Reid M J A, Arinaminpathy N, Bloom A, et al. Building a tuberculosis-free world: the Lancet Commission on tuberculosis. Lancet 2019; 393: 1331-1384.

5 Wells W A, Stallworthy G, Balsara Z. How tuberculosis programs can navigate the world of health insurance. Int J Tuberc Lung Dis 2019; 23: 26-37. 